\title{
Siglecs, Novel Immunotherapy Targets, Potentially Enhance The Effectiveness of Existing Immune Checkpoint Inhibitors in Glioma Immunotherapy
}

This article was published in the following Dove Press journal: OncoTargets and Therapy

\author{
Guan-Zhang Li ${ }^{1, *}$ \\ Ke-Nan Zhang ${ }^{1} *$ * \\ Zheng Wang ${ }^{2}$ \\ Hui-Min $\mathrm{Hu}^{\prime}$ \\ Zhi-Liang Wang' \\ Ruo-Yu Huang ${ }^{\prime}$ \\ Hao-Yu Jiang ${ }^{2}$ \\ You Zhai' \\ Yue-Mei Feng' \\ Yuan-Hao Chang' \\ Ren-Peng $\mathrm{Li}^{2}$ \\ Fan $\mathrm{Wu}{ }^{\prime}$ \\ Fan Zeng (D) \\ Tao Jiang ${ }^{1-4}$ \\ Wei Zhang ${ }^{2}$
}

'Department of Molecular Neuropathology, Beijing Neurosurgical Institute, Capital Medical University, Beijing 100070, People's Republic of

China; ${ }^{2}$ Department of Neurosurgery,

Beijing Tiantan Hospital, Capital Medical

University, Beijing 100070, People's

Republic of China; ${ }^{3}$ Center of Brain

Tumor, Beijing Institute for Brain

Disorders, Beijing 100070, People's

Republic of China; ${ }^{4}$ China National

Clinical Research Center for

Neurological Diseases, Beijing 100070,

People's Republic of China

*These authors contributed equally to this work

Correspondence: Wei Zhang; Tao Jiang Department of Neurosurgery, Beijing

Tiantan Hospital, Capital Medical

University, No. II 9 South Fourth Ring

Road West, Fengtai District, Beijing,

People's Republic of China

$\mathrm{Tel}+86$ I80 I02। 26III

Email zhangwei_vincent@126.com;

taojiang1964@I63.com
Background: Inhibitors of immune checkpoints have shown little effect in clinical trials involving glioma patients. Here, we explored novel targets for use in future treatments. Previous studies showed the sialic acid-binding Ig-like lectin (Siglec) family to have a specific role in immunosuppression. We aimed to study the characteristics and immune function of Siglec family members.

Methods: Transcriptome data from 1024 glioma samples and 1551 glioma single cells were used in our study. Clinical and molecular pathology information was also included. Statistical, bioinformatical methods, and single-cell sequencing analysis were applied to investigate the role of Siglec family members.

Results: Siglecs-5, $-7,-9$, and -16 showed a significant correlation with immunosuppression in glioma. They are typically expressed in higher grade, IDH-wildtype, and mesenchymal subtype gliomas. Siglec- $5,-7$, and -9 had a similar immune function to TIM-3, while Siglec-16 was similar to PD-L1, suppressing tumor immunity via different mechanisms. Joint use of Siglecinhibitors and immune checkpoint inhibitors could prolong the survival of glioma patients.

Conclusion: Siglec-5, $-7,-9$, and -16 suppressed tumor immunity in different ways. Joint usage of inhibitors may be an effective means to improve the efficacy of glioma immunotherapy. Keywords: glioma, Siglec family, immunotherapy, single-cell sequencing analysis, prognosis

\section{Introduction}

Gliomas, the most common and most malignant brain tumors in adults, are highly resistant to traditional treatments. ${ }^{1}$ Despite aggressive treatment approaches, consisting of neurosurgical resection followed by adjuvant chemo- and radiotherapy, prognosis remains poor. ${ }^{2}$ Newly developed immunotherapy approaches may improve outcomes in glioma patients. ${ }^{3,4}$

The 2018 Nobel Prize for Medicine Prize was awarded for work showing that the inhibition of negative immune regulation can be used to treat cancer. Various inhibitors of immune checkpoints have been investigated in research and clinical trials, demonstrating remarkable effectiveness. ${ }^{5-7} \mathrm{PD}-1 / \mathrm{PD}-\mathrm{L} 1$-targeting therapy has been shown to be effective in several tumor types. ${ }^{3,6-9}$ Thus, the US Food and Drug Administration (FDA) has approved the clinical application of immune checkpoint inhibitors in melanoma, breast cancer, renal cancer, and lung cancer. However, PD-1/PD-L1 inhibitors showed little therapeutic effect in glioma. ${ }^{10-12}$ Phase II and phase III clinical trials of durvalumab and nivolumab found no significant improvement in median overall survival. ${ }^{13,14}$ Therefore, it is imperative to explore novel targets to develop effective treatments or for use in joint treatments. 
The sialic acid-binding Ig-like lectins (Siglecs) are a protein family of classical immunoglobulin-like lectins. Under physiological conditions, only 15 human and nine murine Siglec molecules are expressed on the surface of myeloid cells and immune cells mediating immune suppression. ${ }^{15,16}$ Recent research showed that Siglec members are broadly overexpressed in human cancer cells and tumor-infiltrating myeloid cells, ${ }^{17,18}$ and that their distribution is mutually exclusive with that of PD-L1. ${ }^{19}$ In particular, Siglec-10 was identified as the receptor of CD24, delivering a "don't eat me" signal; ${ }^{20}$ it was also discovered on extracellular vesicles inhibiting $\mathrm{T}$ cell activity. ${ }^{21}$ Similarly, Siglec-15 was shown to function as an immune suppressor in colorectal cancer. ${ }^{22}$

However, the relationship between Siglec family members and checkpoints in glioma remained unknown. Here, we performed an integrative investigation of Siglecs in 325 glioma samples and validated the results using The Cancer Genome Atlas (TCGA) database and single-cell RNA sequencing (RNA-seq) data from the GSE89567 dataset. This is the first integrative study describing the molecular and clinical characteristics of Siglecs in glioma and demonstrating the potential use of Siglec inhibitors in joint treatment with immune checkpoint inhibitors.

\section{Materials and Methods}

\section{Patients and Samples}

Sample collection and data analyses were approved by the Beijing Tiantan Hospital institutional review board (IRB), and written informed consent was obtained from each patient. Samples with more than $80 \%$ tumor cells were selected for transcriptome sequencing on an Illumina HiSeq platform. Each sample was diagnosed by two independent neuropathologists. Overall survival was calculated from the date of diagnosis to the date of death or last follow-up. TCGA RNA-seq data were downloaded from the official website (https://cancergen ome.nih.gov). Single-cell sequencing data were obtained from GSE89567 on the GEO website.

\section{Gene Set Variation Analysis (GSVA)}

GSVA analysis was performed with the GSVA package. ${ }^{23}$ The list of gene ontology terms was obtained from the GSEA Web portal (http://software.broadinstitute.org/gsea/msigdb/ index.jsp). The analysis was performed with default parameters. Relationships between genes and biological functions were determined using Pearson correlation analysis.

\section{T-Distributed Stochastic Neighbor Embedding (T-SNE) Analysis}

The t-SNE analysis was performed with the Rtsne package. Perplexity was set to 20. Identification of cell types used specific cell markers obtained from the official CellMarker website (http://biocc.hrbmu.edu.cn/CellMarker/).

\section{Statistical Analysis}

Statistical analyses and visualization were performed in R 3.5.0, SPSS software 25.0, and Microsoft Office 2016. SPSS statistical software was used for the Cox regression analysis. Radar charts were created in Microsoft Office 2016. Other analyses were performed with $\mathrm{R}$ packages, including ggplot $2, p R O C,{ }^{24}$ and pheatmap. The log-rank test was used in Kaplan-Meier survival analysis. A p-value less than 0.05 was considered statistically significant.

\section{Results}

Siglec-5, $-7,-9$ and -16 are Independent Prognostic Factors Associated with Malignant Progression in Glioma

The expression landscape of Siglec family members in glioma showed that most members were differentially expressed in both the CGGA and TCGA databases, except Siglec-6 (Figure 1A and B). The multivariate Cox analysis revealed that Siglec-5, $-7,-9$ and -16 are independent to clinical and molecular pathological factors in both databases (Figure 1C). In addition, Siglec-5, $-7,-9$ and -16 showed higher expression levels in high-grade gliomas (Figure 1D and E), suggesting that these Siglecs are associated with tumor progression in glioma.

\section{Siglec-5, $-7,-9$, and -16 are Enriched in IDH-Wildtype and MGMT Promotor Unmethylated Glioma}

IDH mutation and MGMT promotor methylation status are the two most significant prognostic biomarkers for glioma. ${ }^{25}$ Therefore, we explored the relationships between IDH mutation and MGMT promotor methylation status and the expression of Siglec-5, -7, -9 and -16 . As shown in the column diagrams, all four Siglec members showed significantly higher expression in the IDH-wildtype and MGMT promotor unmethylated groups (Figure 2A and B, Figure S1A and B). Subsequent receiver operating characteristic (ROC) curve analysis showed that Siglec-5, $-7,-9$ and -16 were specifically enriched in IDH-wildtype gliomas (Figure S2A and B). 
A

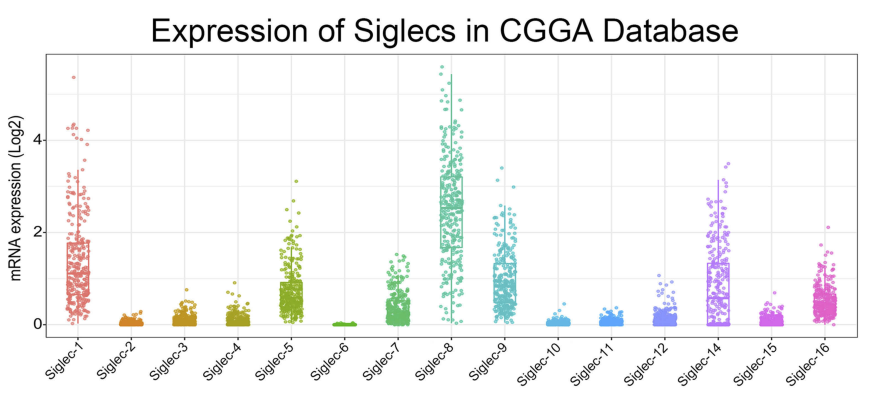

B

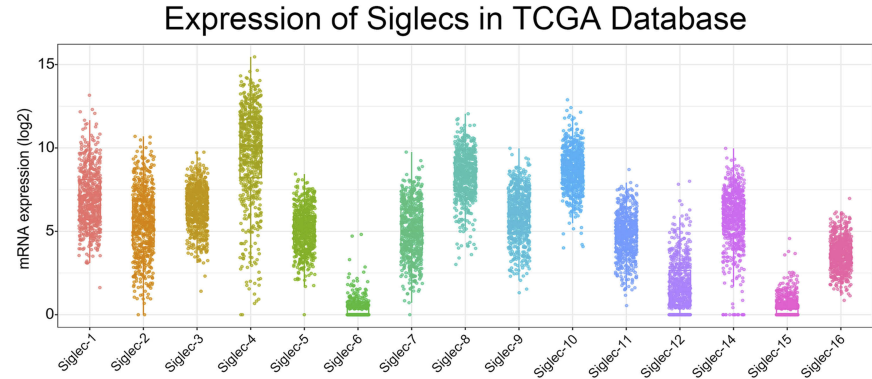

C Multivariate COX analysis of each siglecs

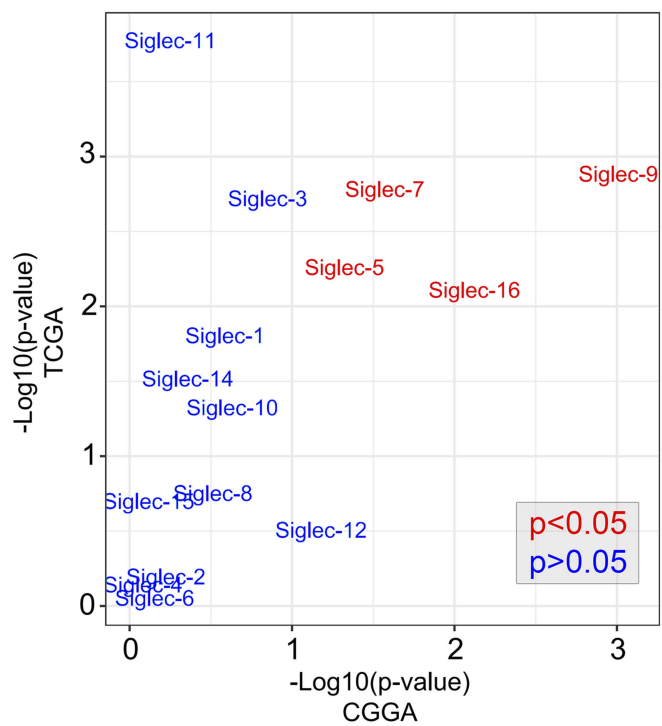

E
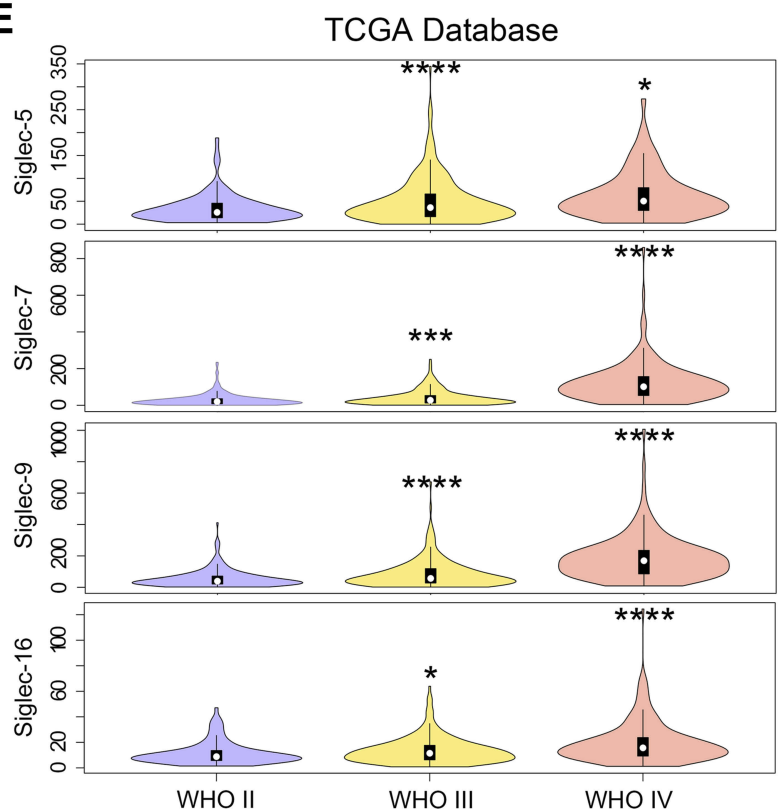

D
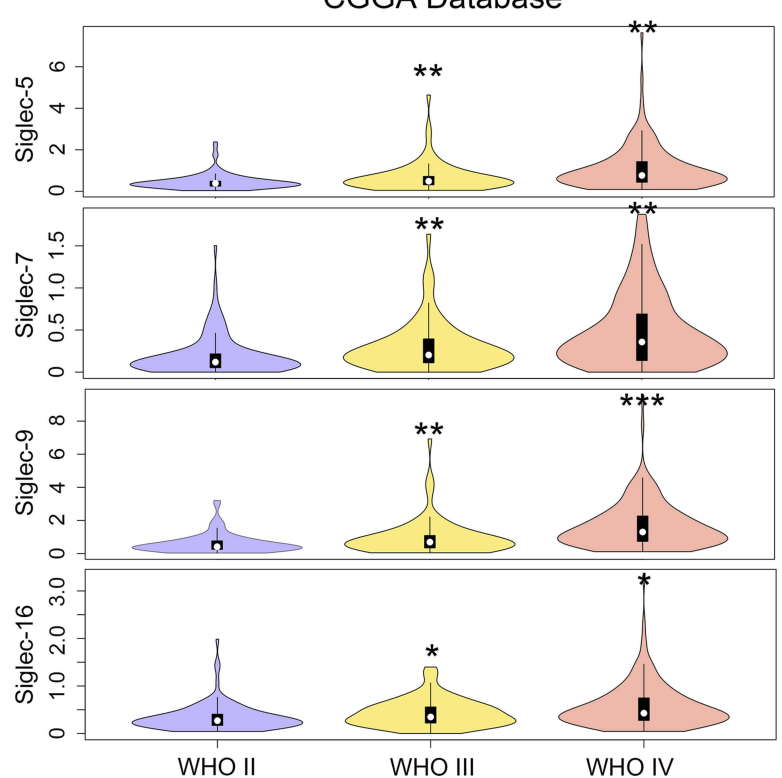

Figure I Expression landscape of Siglec family members in glioma. (A, B) Transcriptome expression map of Siglecs in the CGGA and TCGA database. (C) P-values for multivariate Cox analysis of each Siglec member in the CGGA and TCGA databases. Variables in the multivariate Cox analysis included Siglec member, WHO grade, age, and IDH mutation status. Red font indicates an independent prognostic factor. (D, E) Violin plot showing the expression of Siglecs in each WHO grade glioma according to the CGGA and TCGA databases. ${ }^{*} \mathrm{p}<0.05,{ }^{*} \mathrm{p}<0.0 \mathrm{I},{ }^{* * *} \mathrm{p}<0.00 \mathrm{I}, * * * * \mathrm{p}<0.000 \mathrm{I}$.

All these results were mutually verified using the CGGA and TCGA databases.

\section{Siglec-5, $-7,-9$, and -16 are Potential Markers for the Mesenchymal Molecular Subtype}

Specific enrichment in the mesenchymal molecular subtype is an important feature of immune checkpoints. ${ }^{26}$ Thus, we explored the distribution of four Siglec members in different molecular subtypes defined by the TCGA network. $^{27}$ As shown in Figure 3, four Siglec family members had higher expression in the mesenchymal subtype. Accordingly, the enrichment of Siglec-5, $-7,-9$, and -16 in the mesenchymal subtype were also specifically validated by ROC curve analysis (Figure S2E and F). The specific expression pattern was found in both the CGGA and TCGA databases, indicating a potential immune-related feature of Siglecs. 
A

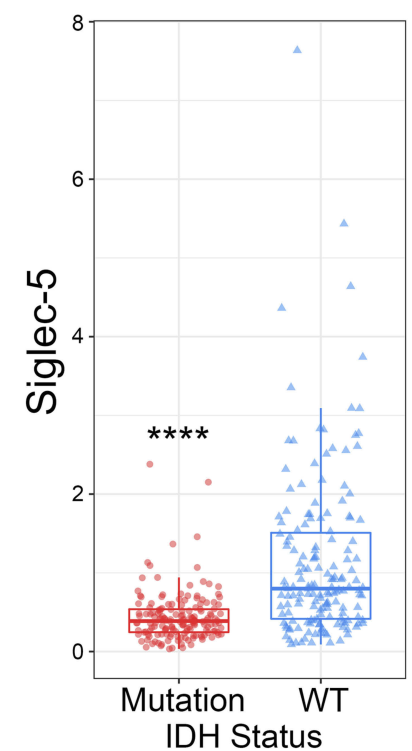

B

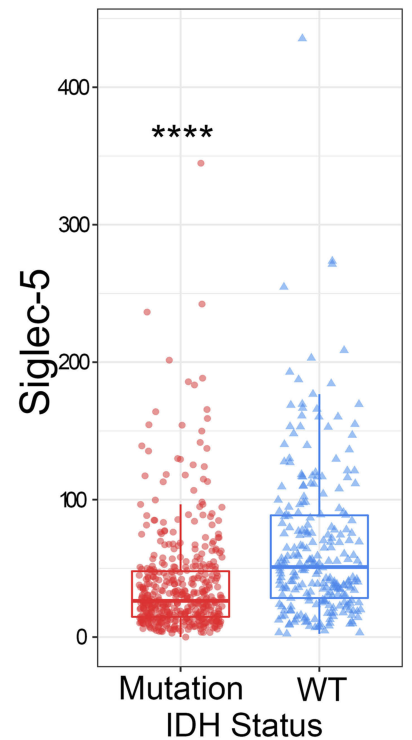

Siglecs and IDH1 mutation in CGGA Database
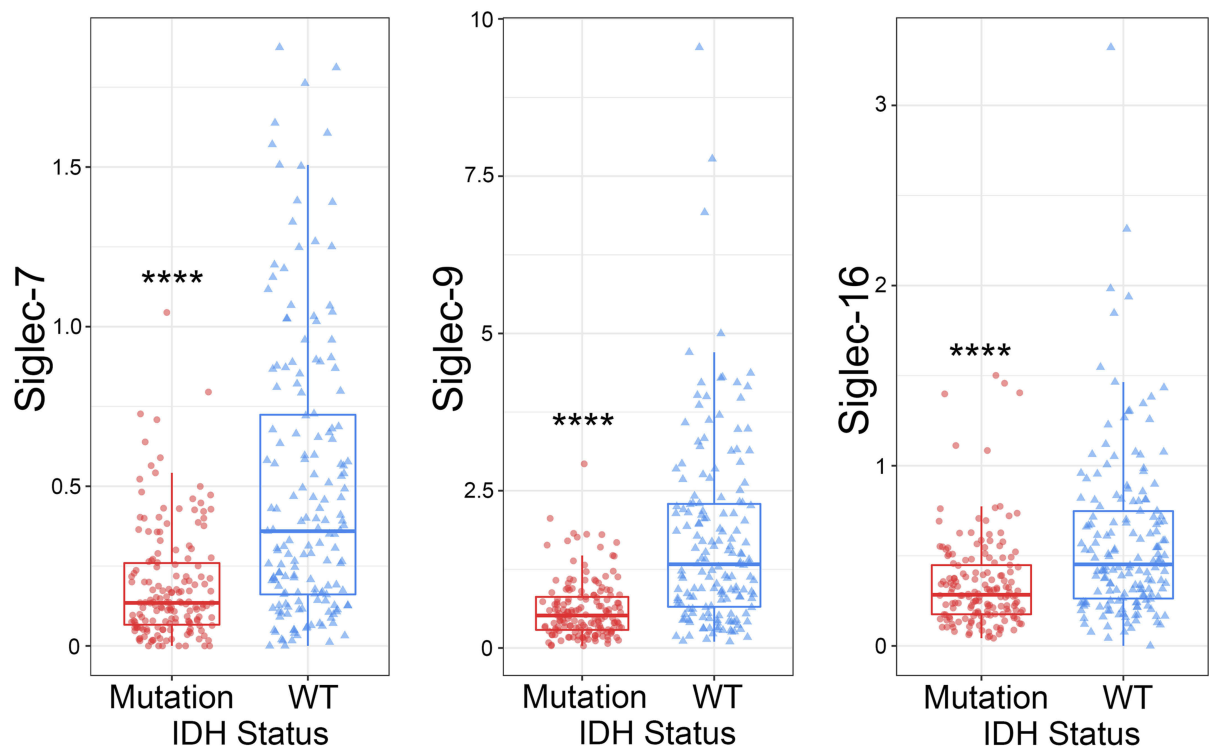

Siglecs and IDH1 mutation in TCGA Database
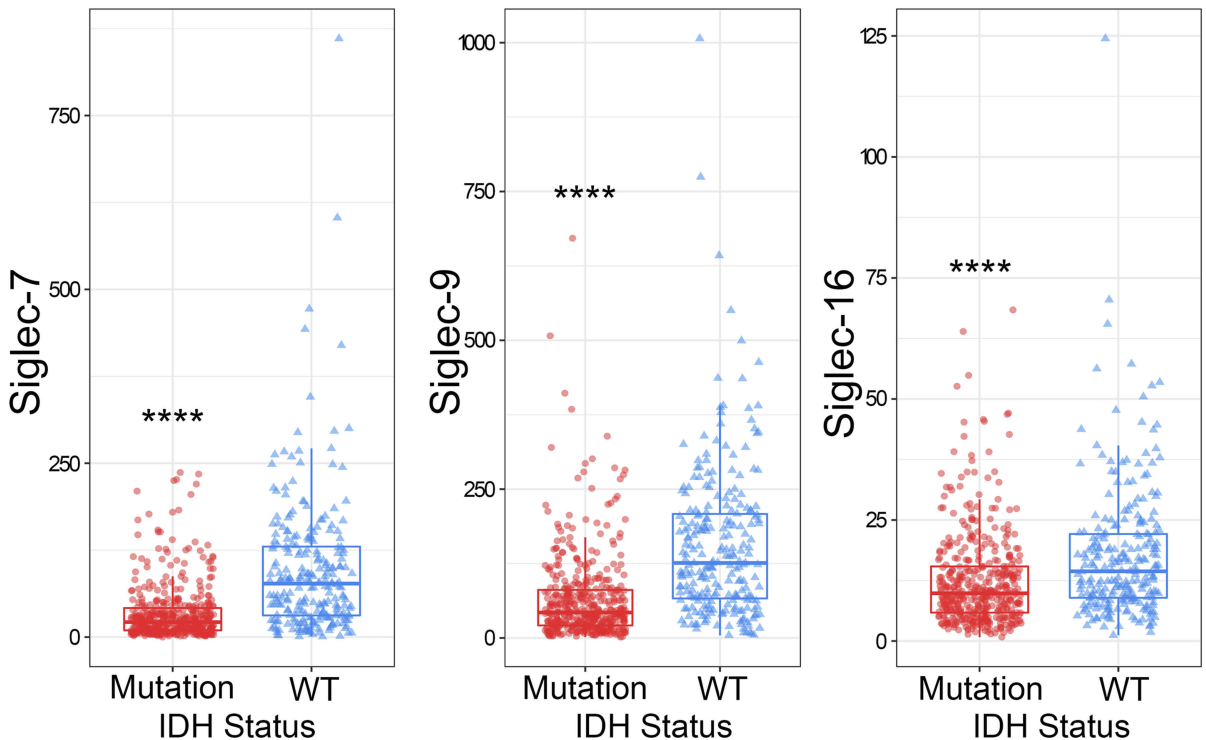

Figure 2 Siglec-5, $-7,-9$, and -16 are highly expressed in IDH-wild-type gliomas. The expression of Siglec-5, $-7,-9$, and -16 was higher in IDH-wildtype gliomas than in IDH-mutated gliomas, according to the CGGA (A) and TCGA (B) databases. $* * * * 0<0.0001$.

Siglec-5, $-7,-9$, and -16 are Closely Related to Immune Functions in Glioma

Unsupervised clustering analysis was used to determine the expression patterns of Siglecs and known immune checkpoints. Siglec-5, -7 , and -9 had similar expression patterns, whereas that of Siglec-16 was quite different (Figure 4A and C). We further analyzed the correlation of the four Siglec family members and 4436 biological functions in 14 functional classes to investigate related biological processes. All four Siglecs were positively correlated with approximately $99 \%$ of immune functions (Figure 4B and D). All related biological functions are listed in Supplementary Table 1. Highly consistent results were observed with the CGGA and TCGA databases, indicating that Siglec-5, -7 and -9 , and -16 are commonly immune-related, but in slightly different ways from each other.

\section{Siglec-5, -7, -9, and - 16 Synergize with Different Immune Checkpoints}

To explore the distinctions between the immune-related functions of Siglec-5, $-7,-9$, and -16 , we performed 
A

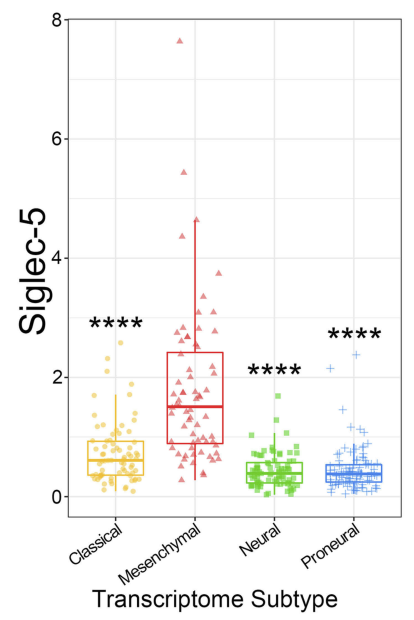

B

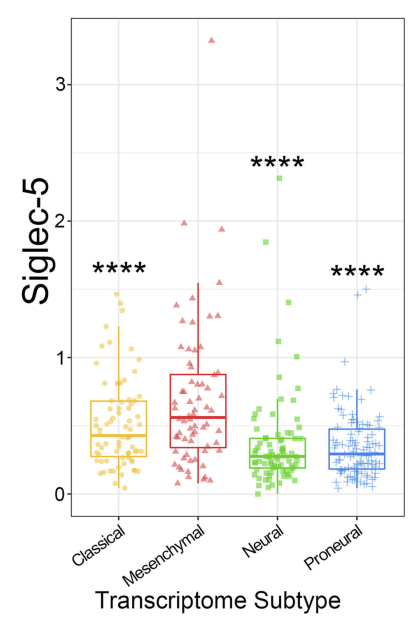

Siglecs and Transcriptome Subtype in CGGA Database
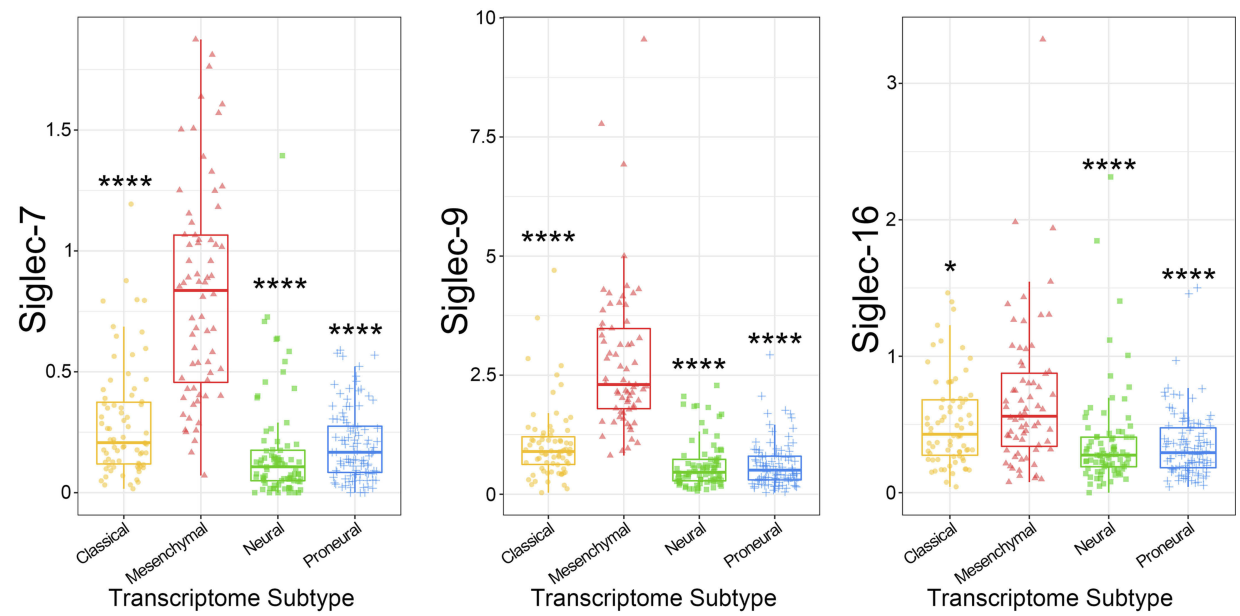

Siglecs and Transcriptome Subtype in TCGA Database
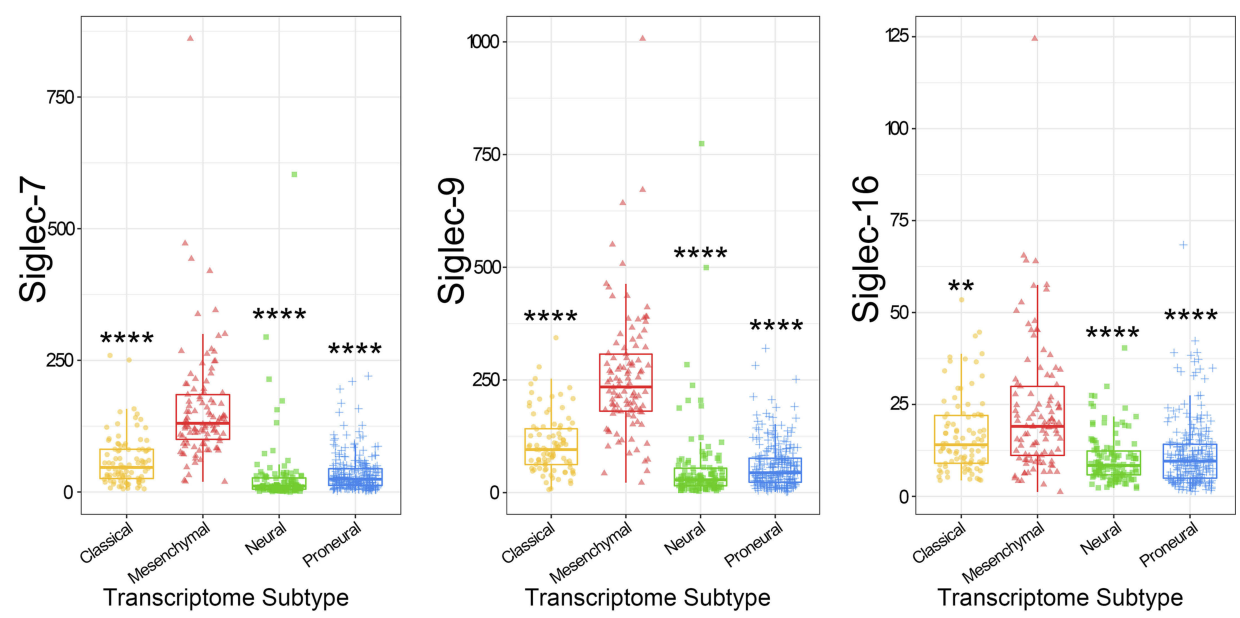

Figure 3 Siglec-5, $-7,-9$, and -16 are highly enriched in mesenchymal subtype. The expression of Siglec-5, $-7,-9$, and -16 was higher in mesenchymal subtype gliomas than others, according to the CGGA (A) and TCGA (B) databases. ${ }^{*} p<0.05, * * p<0.01, * * * * p<0.0001$.

t-SNE analysis for the expression levels of Siglecs and known immune checkpoints. There were similarities among Siglec-5, -7, and -9, TIM-3, galectin-9, B7-1, and CD40; and Siglec -16 and PD-L1, PD-1, CTLA4, B7-2, CD28, and HVEM had similar roles in both the CGGA and TCGA databases (Figure 5A and C). To further validate the relationship between Siglecs and immune functions, we calculated the correlation with inflammatory activities. As shown in Figure 5B and D, Siglec-5, -7, and -9, TIM-3, and galectin-9 had almost the same inflammatory activation status. Siglec-16, B7-2, PD1, PD-L1, and CTLA4 were also similar to each other (Figure 5B and D).

\section{Single-Cell Sequencing Reveals the Difference Between Siglec-5, -7, and -9 and Siglec -16}

The development of single-cell sequencing technology enabled a deeper understanding of the heterogeneity among cells. ${ }^{28,29}$ We found that Siglec-5, -7 , and -9 expression was mutually exclusive in cells in WHO grade II gliomas. However, the expression pattern changed in higher-grade gliomas (WHO III and WHO IV), where different Siglecs were simultaneously expressed on the same cells (Figure 6A). However, Siglec-16 was expressed exclusively to Siglec-5, -7 , and -9 in glioma cells of all grades (Figure 6A). Next, we studied the 
A Biological Function Landscape in CGGA Database

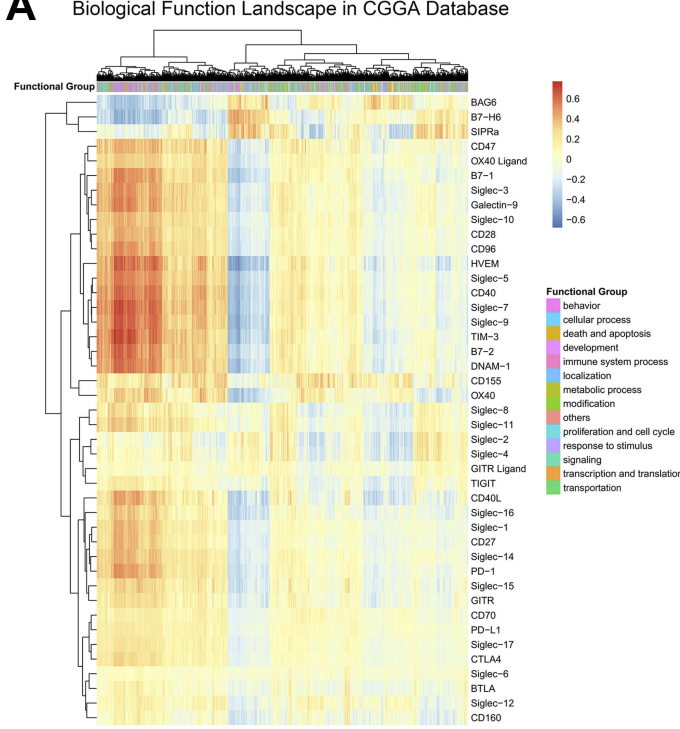

C Biological Function Landscape in TCGA Database

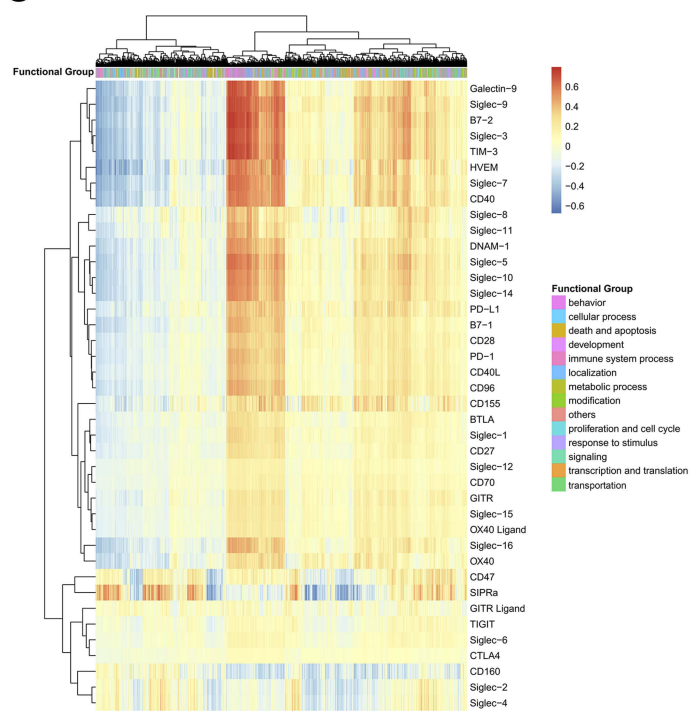

B

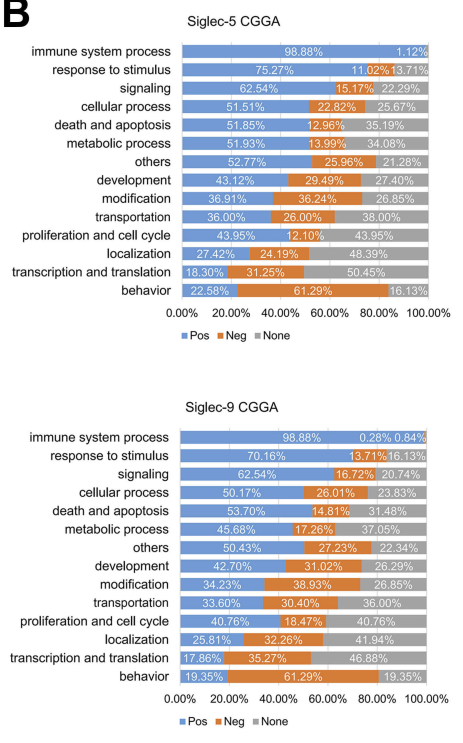

D

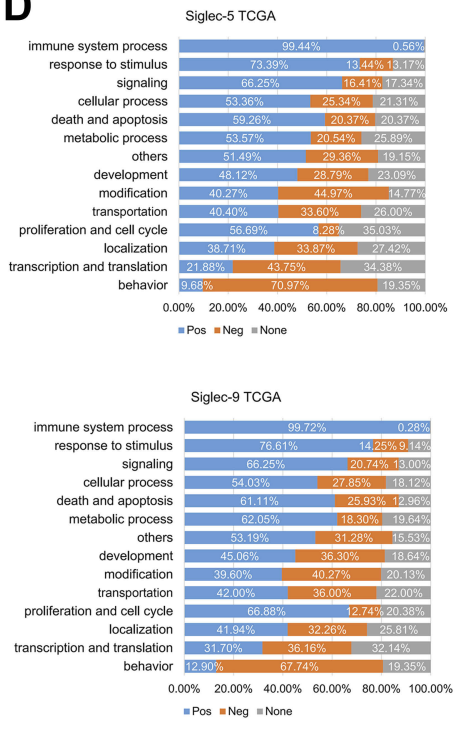

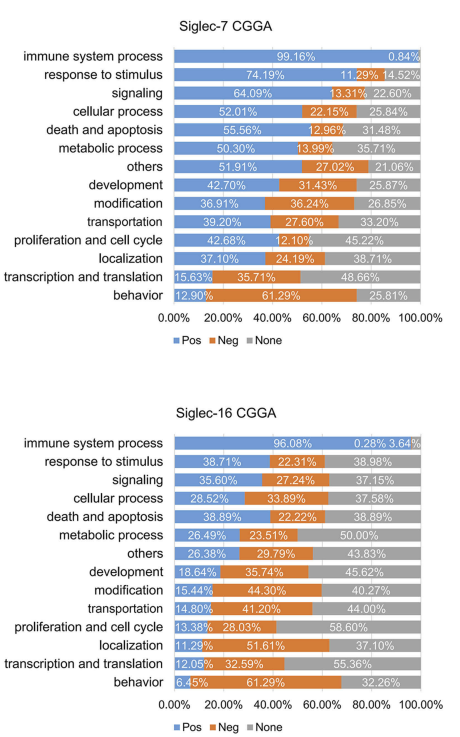

= Pos $=$ Neg $=$ None
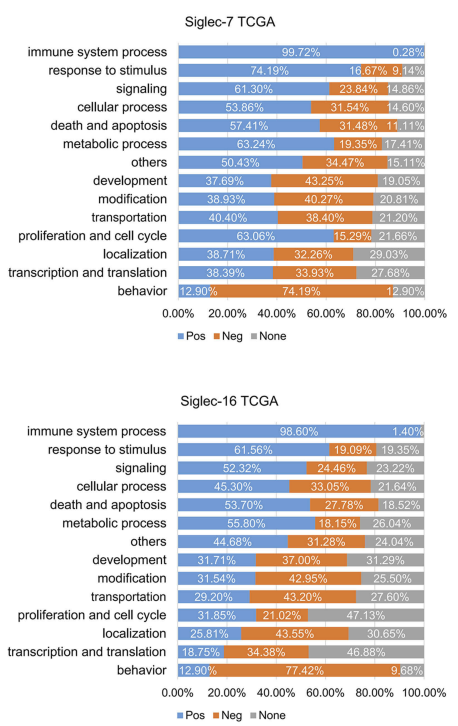

Figure 4 Siglec-5, $-7,-9$, and -16 are closely related to immune functions in glioma. (A, C) Functional heatmaps of Siglecs and immune checkpoints in the CGGA and TCGA databases. (B, D) Proportion of Siglec-related biological functions in each function class. Blue parts represent positive correlation; orange represents negative correlation; gray indicates no significant correlation.

relationships between Siglec-5, $-7,-9$, and -16 and immune checkpoints. Siglec-16 expression was exclusive to PD-1, PD-L1, CTLA4, CD28, and HVEM (Figure 6B), whereas no exclusive pattern was found for Siglec-5, -7, and -9 and TIM-3, galectin-9, B7-1, and CD40 (Figure S3). Interestingly, spatial mapping analysis revealed that Siglec-5, -7 , and -9 , but not Siglec-16, gradually transferred from tumor cells to immune cells with increasing malignancy (Figure 6C and D). Further subdividing the Siglec-expressing immune cells, we found that macrophages and tumorassociated macrophages (TAMs) accounted for a major portion (Figure 6E). In an open-access single-cell sequencing database, the main distribution was also in macrophages, providing an external validation of our results (Figure S4) ${ }^{30}$

\section{Joint Usage of Siglec Inhibitor and Immune Checkpoint Inhibitor May Benefit Glioma Patients}

To explore the potential effects of joint usage of a Siglec inhibitor and immune checkpoint inhibitor on survival, a combined prognostic analysis was performed. In the 


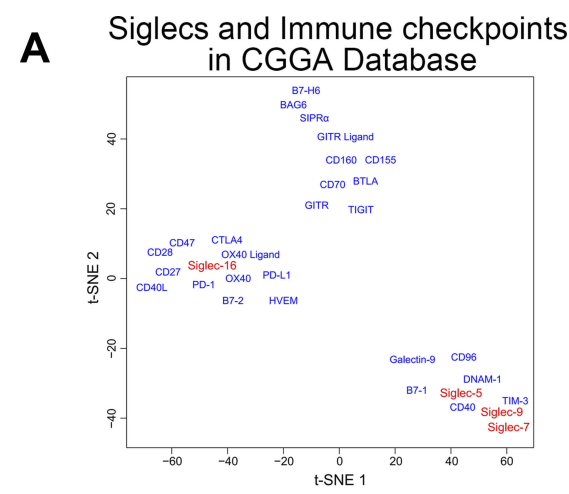

Siglecs and Immune checkpoints

C

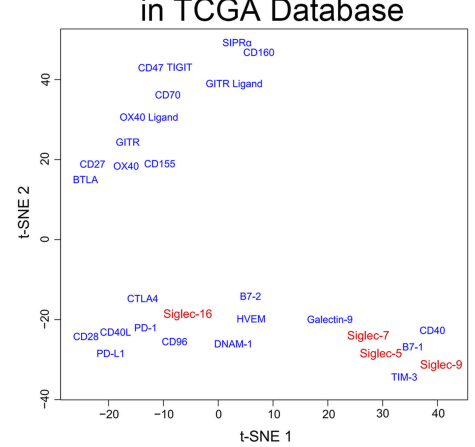

B
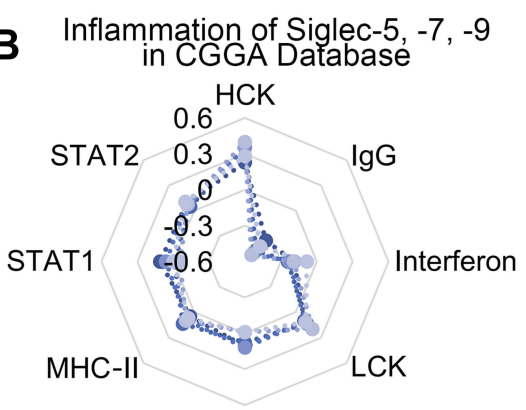

MHC-I

...... SIGLEC5 …... SIGLEC7 …... SIGLEC9

...... TIM-3 …... Galectin-9

D Inflammation of Siglec-5, -7, -9

in TCGA Database

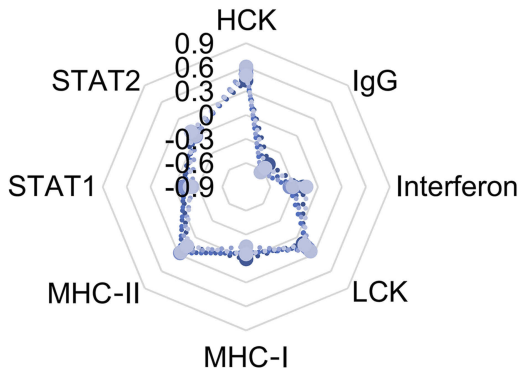

Inflammation of Siglec-16

in CGGA Database

$0.6^{\mathrm{HCK}}$

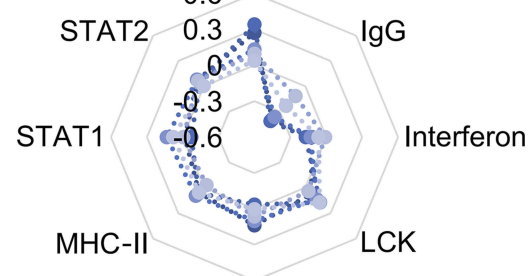

MHC-I

$\cdots \bullet \cdots$ SIGLEC16 $\quad \cdots \cdot \cdots$ B7-2 $\quad \cdots \cdot \cdots$ PD-1

…...PD-L1 …... CTLA4

Inflammation of Siglec-16

in TCGA Database

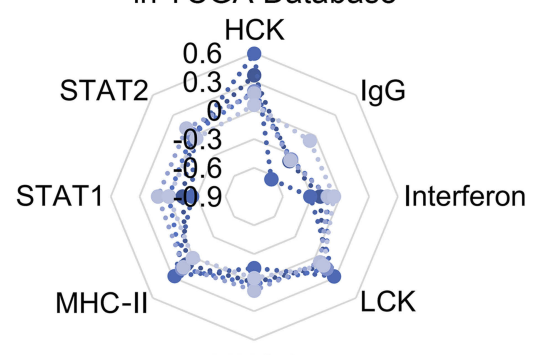

MHC-I

Figure 5 Siglec-5, $-7,-9$, and -16 and immune checkpoints share biological functions. (A, C) Functional distribution of Siglec-5, $-7,-9$, and -16 and immune checkpoints by t-SNE analysis in the CGGA and TCGA databases. (B, D) Radar diagrams of the correlation between Siglec-5, $-7,-9$, and -16 and inflammatory activity. The coordinates in the figure are the $R$ values from Pearson correlation analysis of the Siglecs and inflammatory response scores.

former retrospective survival analysis, patients with overexpression of immune checkpoints were regarded as applicable target patients. The Kaplan-Meier curves revealed that patients with low expression of both Siglecs and synergistic immune checkpoints had better survival than patients in the other three groups, according to both the CGGA and TCGA databases (Figure 7). All these results indicated a better prognosis in patients under combined treatment compared with those receiving single immune checkpoint inhibitor therapy, indicating an enhancer role for Siglec inhibitors in the combined treatment.

\section{Discussion}

Owing to its great success in the treatment of non-smallcell lung carcinoma, acute lymphocytic leukemia, etc., tumor immunotherapy has flourished in the past few years. ${ }^{6,7,31,32}$ However, the immune checkpoint inhibitors often lose efficacy gradually over time, eventually leading to failure of the treatment. ${ }^{10,12,33}$ A series of studies confirmed that the PD-1/PD-L1 pathway was responsible for immunosuppressive status in less than $40 \%$ of solid tumors. ${ }^{31,34}$ Thus, it is necessary to screen out new targets for immunotherapy. The Siglec family of novel immune- regulators has attracted research attention in this regard. ${ }^{16}$ Recently, Siglec-15 was screened out as a new therapeutic target by a high-throughput functional screening system (TCAA), expressed exclusively in PD-1/PD- L1 in tumors. $^{19}$ In glioma, Santegoets et al found that the Siglecs were highly expressed in tumor cells and immune cells. ${ }^{35}$ Based on the above studies, we hypothesized that Siglecs might significantly regulate tumor immunity in glioma.

In this work, we found a specific expression pattern of Siglec family members in 1024 gliomas and confirmed Siglec-5, $-7,-9$, and -16 as independent prognostic factors. Their correlations with IDH mutation, MGMT promotor methylation status, and mesenchymal subtype suggested their potential as biomarkers. Although the correlation with MGMT promotor methylation status was not significant, this may have been due to missing data. Further functional analysis revealed the immune-related features of these Siglecs, including leukocyte activation, natural killer cell differentiation, positive regulation of macrophage activation, and regulation of $\mathrm{T}$ cell chemotaxis, indicating that they deserve further future research. Siglec-5, -7 , and -9 were also identified in a study by 
A

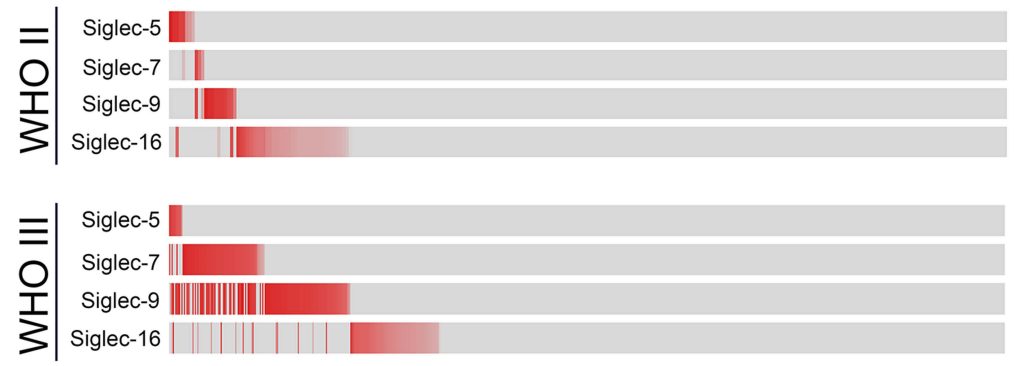

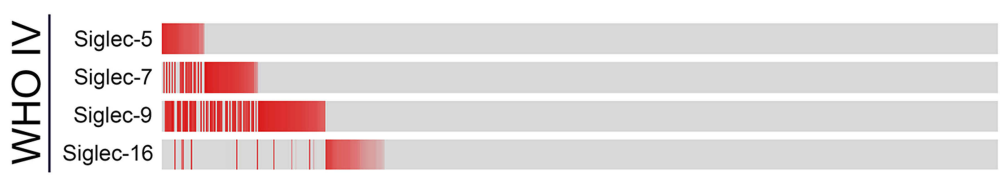

C
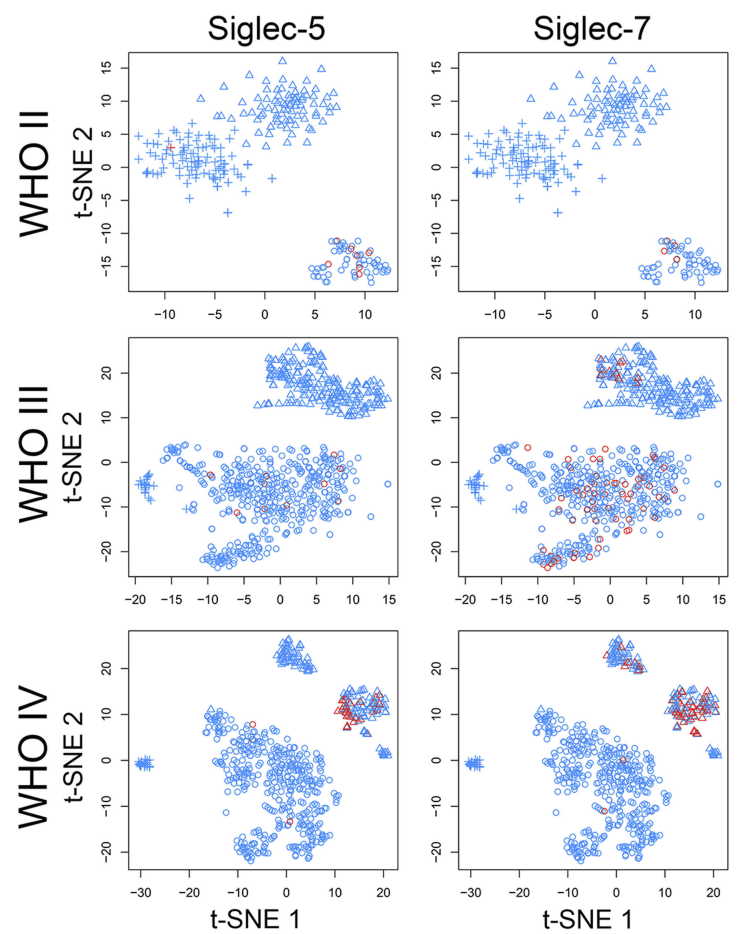

D Siglecs and cell types in glioma patients

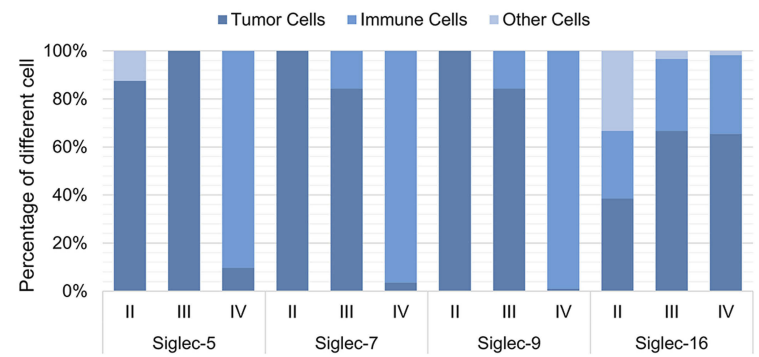

B

GSE89567
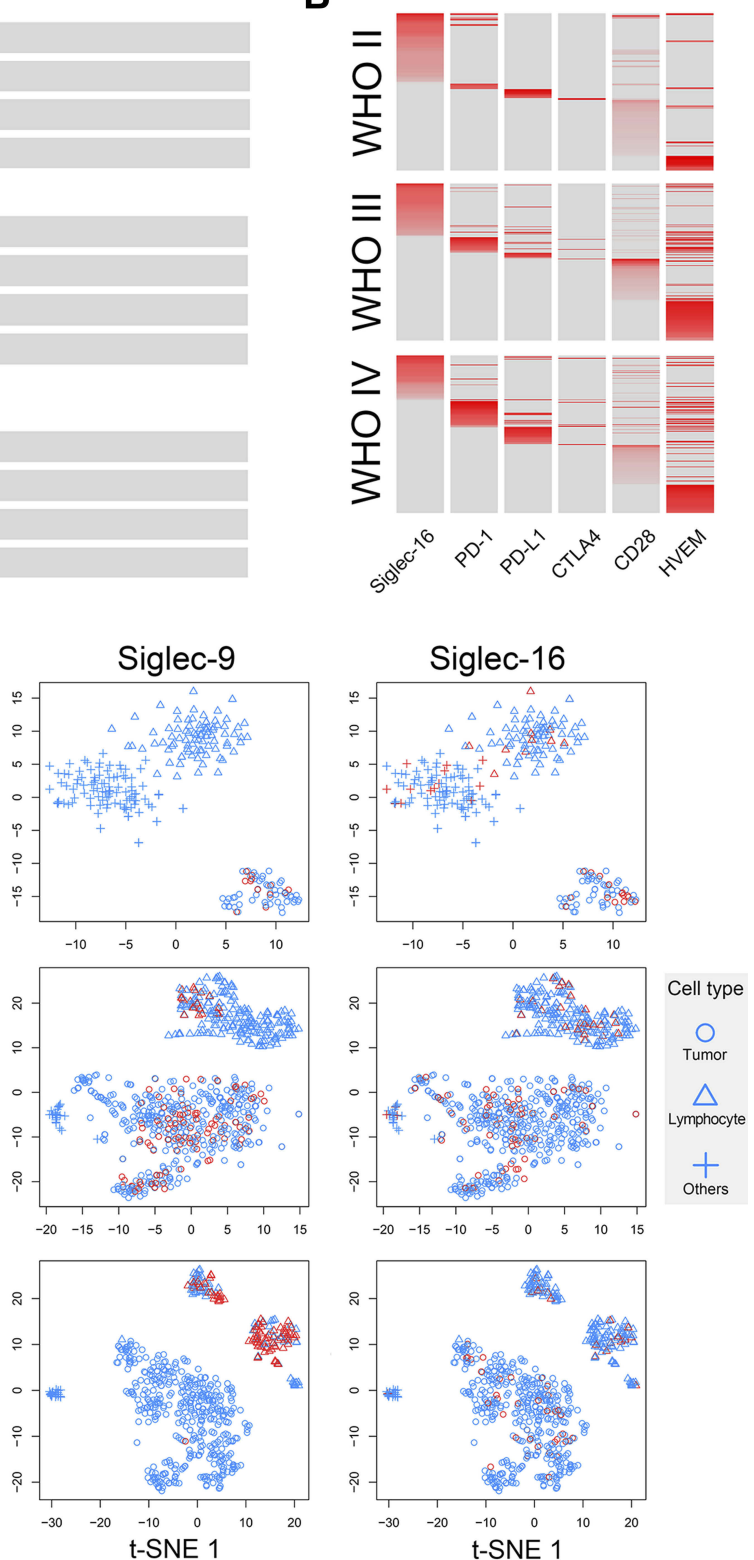

\section{E Siglecs in immune cell types}

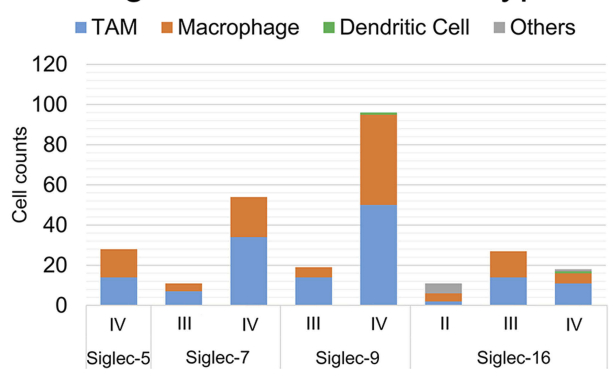

Figure 6 Immunological characteristics are different in Siglec-5, -7, -9 , and -16 . (A) Expression of Siglec-5, $-7,-9$, and -16 in glioma cells of each WHO grade. The expression level is represented by the shade of red. Gray indicates no expression. (B) Expression of Siglec-16 and immune checkpoints in glioma cells of each WHO grade. The expression level is represented by the shade of red. Gray indicates no expression. (C) Spatial mapping analysis of each WHO grade glioma. Red indicates that Siglec was expressed in the cell. Cells without Siglec expression are colored blue. (D) The distribution proportion of Siglec members in each cell type. (E) Number of immune cells with Siglec members expressed. 
A

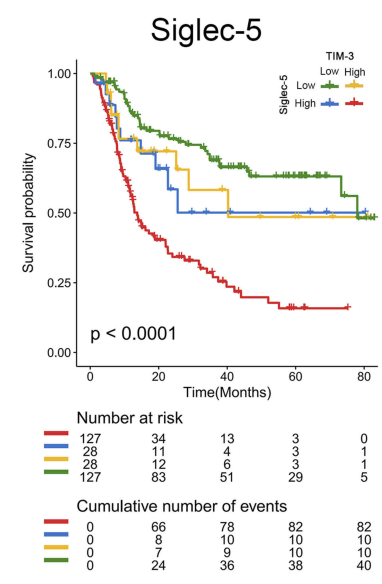

B

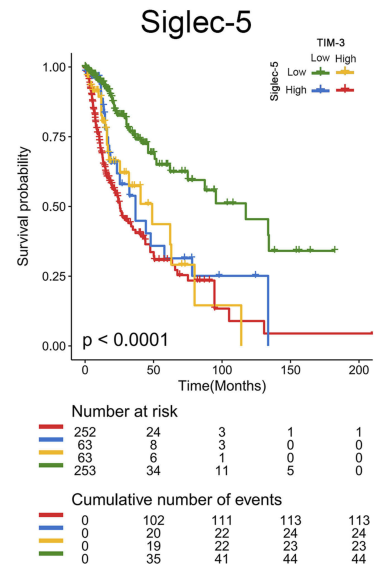

Survival analysis of Siglecs in CGGA Database

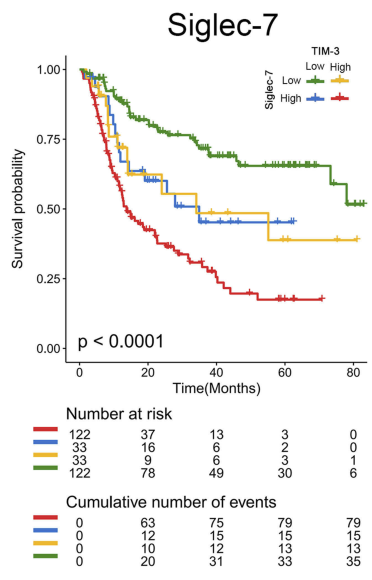

Siglec-9
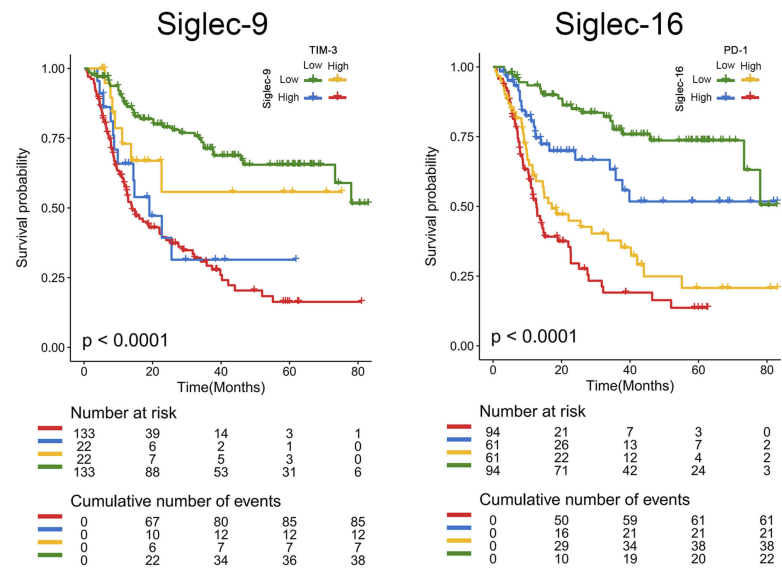

\section{Survival analysis of Siglecs in TCGA Database}
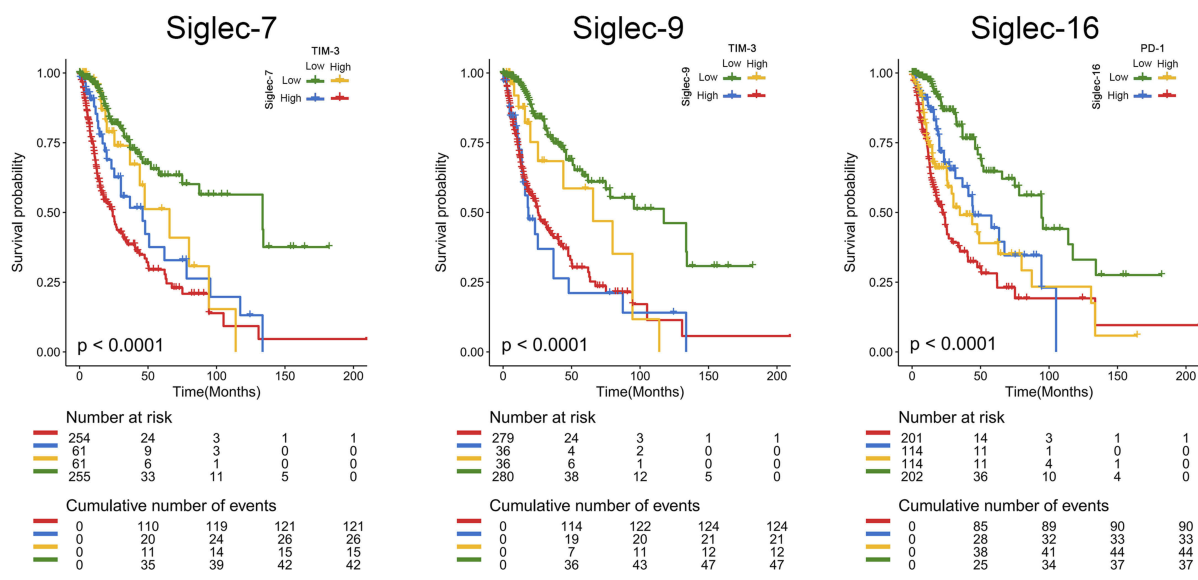

Figure 7 Joint usage of Siglec inhibitor and corresponding immune checkpoint inhibitor may benefit glioma patients. (A, B) Kaplan-Meier curves of patients with different expression patterns of Siglec members and immune checkpoints in the CGGA and TCGA database. Patients with low expression of Siglec members and corresponding immune checkpoints had the longest overall survival time. The log-rank test was used for survival analysis.

Santegoets ${ }^{35}$ and were shown to have similar immune functions to TIM- $3,{ }^{36}$ whereas Siglec-16 was different, with a function complementary to that of PD-1/PD-L1. ${ }^{26}$

Single-cell sequencing analysis revealed that Siglec-5, -7 , -9 , and -16 were expressed synergistically with TIM-3, mainly in macrophages and TAMs; this has been reported in leukemia, glioma, etc. ${ }^{17,19,35}$ Interestingly, we first reported the expression shift of Siglec-5, -7 , and -9 from tumor cells to macrophages with increasing tumor malignancy, possibly owing to trogocytosis of macrophages. ${ }^{37}$ This may induce immune escape in high-grade gliomas. However, no such expression shift was detected in Siglec-16 expressing cells, indicating the selectivity of macrophage trogocytosis. Siglec16 was expressed exclusively with PD-1, PD-L1, and CTLA4 in gliomas, similar to the reported function of Siglec-15. ${ }^{19}$
In the survival analysis, patients were divided into four groups based on the expression of Siglec members and immune checkpoints, and their Kaplan-Meier curves were compared, as in previously published research. ${ }^{38}$ The results indicated that patients with low expression of both Siglecs and the corresponding immune checkpoints had a better prognosis than others, suggesting that the joint usage of a Siglec inhibitor and corresponding immune checkpoint inhibitors may prolong the overall survival of glioma patients.

\section{Conclusions}

In conclusion, Siglec family members showed specific expression patterns in gliomas and had diverse roles in the immunosuppression process, interacting with different 
immune checkpoints. This indicates the high potential of a combination of Siglec inhibitors and immune checkpoint inhibitors to solve the current problems of immunotherapy in glioma.

\section{Ethics Approval and Informed Consent}

Sample collection and data analyses were approved by Beijing Tiantan Hospital IRB. Written informed consent was obtained from each participant. The study was conducted in accordance with the Declaration of Helsinki.

\section{Data Availability}

The datasets used and analyzed in the current study are available from the corresponding author on reasonable request.

\section{Acknowledgments}

This work was supported by grants from the National Natural Science Foundation of China (No. 81672479, 81802994), National Natural Science Foundation of China/Research Grants Council Joint Research Scheme (81761168038), and Beijing Municipal Administration of Hospitals' Mission Plan (SML20180501). We thank Ms. Shuqing Sun and Hua Huang for tissue sample collection and clinical data retrieval. Guanzhang $\mathrm{Li}$ and Kenan Zhang are co-first authors for this study.

\section{Author Contributions}

All authors made substantial contributions to conception and design, acquisition of data, or analysis and interpretation of data; took part in drafting the article or revising it critically for important intellectual content; gave final approval of the version to be published; and agree to be accountable for all aspects of the work.

\section{Disclosure}

The authors report no conflicts of interest in this work.

\section{References}

1. Jiang T, Mao Y, Ma W, et al. CGCG clinical practice guidelines for the management of adult diffuse gliomas. Cancer Lett. 2016;375:263-273. doi:10.1016/j.canlet.2016.01.024.

2. Stupp R, Mason WP, van den Bent MJ, et al. Radiotherapy plus concomitant and adjuvant temozolomide for glioblastoma. $N$ Engl J Med. 2005;352:987-996. doi:10.1056/NEJMoa043330.

3. Topalian SL, Hodi FS, Brahmer JR, et al. Safety, activity, and immune correlates of anti-PD-1 antibody in cancer. $N$ Engl $J$ Med. 2012;366:2443-2454. doi:10.1056/NEJMoa1200690.
4. Xue S, Hu M, Iyer V, Yu J. Blocking the PD-1/PD-L1 pathway in glioma: a potential new treatment strategy. J Hematol Oncol. 2017;10:81. doi:10.1186/s13045-017-0455-6.

5. Hodi FS, O'Day SJ, McDermott DF, et al. Improved survival with ipilimumab in patients with metastatic melanoma. $N$ Engl $\mathrm{J} \mathrm{Med}$. 2010;363:711-723. doi:10.1056/NEJMoa1003466.

6. Brower V. Anti-PD-L1 antibody active in metastatic bladder cancer. Lancet Oncol. 2015;16:e11. doi:10.1016/S1470-2045(14)71167-2.

7. Herbst RS, Soria JC, Kowanetz M, et al. Predictive correlates of response to the anti-PD-L1 antibody MPDL3280A in cancer patients. Nature. 2014;515:563-567. doi:10.1038/nature14011.

8. Brahmer JR, Tykodi SS, Chow LQ, et al. Safety and activity of anti-PD-L1 antibody in patients with advanced cancer. $N$ Engl J Med. 2012;366:2455-2465. doi:10.1056/NEJMoa1200694.

9. Pardoll DM. The blockade of immune checkpoints in cancer immunotherapy. Nat Rev Cancer. 2012;12:252-264. doi:10.1038/nrc3239.

10. Kurz SC, Cabrera LP, Hastie D, et al. PD-1 inhibition has only limited clinical benefit in patients with recurrent high-grade glioma. Neurology. 2018;91:e1355-e1359. doi:10.1212/WNL.000000000000 6283.

11. PD-1 blockade in GBM: uncovering RESPONSE CLUES. Cancer Discov. 2019. doi:10.1158/2159-8290.CD-ND2019-001.

12. Lim M, Xia Y, Bettegowda C, Weller M. Current state of immunotherapy for glioblastoma. Nat Rev Clin Oncol. 2018;15:422-442. doi:10.1038/s41571-018-0003-5.

13. Reardona DA, Omuroa A, Brandes AA, et al. Randomized phase 3 study evaluating the efficacy and safety of nivolumab Vs bevacizumab in patients with recurrent glioblastoma: checkmate 143. NeuroOncology. 2017;19:21. doi:10.1093/neuonc/nox036.071

14. Reardon DA, Kaley TJ, Dietrich J, et al. Phase 2 study to evaluate safety and efficacy of MEDI4736 (durvalumab [DUR]) in glioblastoma (GBM) patients: an update. J Clin Oncol. 2017;35:2042. doi:10.1200/JCO.2017.35.15_suppl.2042.

15. Pillai S, Netravali IA, Cariappa A, Mattoo H. Siglecs and immune regulation. Annu Rev Immunol. 2012;30:357-392. doi:10.1146/ annurev-immunol-020711-075018.

16. Pearce OM, Laubli H. Sialic acids in cancer biology and immunity. Glycobiology. 2016;26:111-128. doi:10.1093/glycob/ cwv097.

17. Chang J, Peng H, Shaffer BC, et al. Siglec-6 on chronic lymphocytic leukemia cells is a target for post-allogeneic hematopoietic stem cell transplantation antibodies. Cancer Immunol Res. 2018;6:1008-1013. doi:10.1158/2326-6066.CIR-18-0102.

18. Laubli H, Pearce OM, Schwarz F, et al. Engagement of myelomonocytic Siglecs by tumor-associated ligands modulates the innate immune response to cancer. Proc Natl Acad Sci U S A. 2014;111:14211-14216. doi:10.1073/pnas.1409580111.

19. Wang J, Sun J, Liu LN, et al. Siglec-15 as an immune suppressor and potential target for normalization cancer immunotherapy. Nat Med. 2019;25:656-666. doi:10.1038/s41591-019-0374-x.

20. Barkal AA, Brewer RE, Markovic M, et al. CD24 signalling through macrophage Siglec-10 is a target for cancer immunotherapy. Nature. 2019;572:392-+. doi:10.1038/s41586-019-1456-0.

21. Li Y, Zhou J, Zhuo Q, et al. Malignant ascite-derived extracellular vesicles inhibit $\mathrm{T}$ cell activity by upregulating Siglec-10 expression. Cancer Manag Res. 2019;11:7123-7134. doi:10.2147/CMAR. S210568.

22. Wang J, Sun JW, Liu LN, et al. Siglec-15 as an immune suppressor and potential target for normalization cancer immunotherapy. Nat Med. 2019;25:656-+. doi:10.1038/s41591-019-0374-x.

23. Hanzelmann S, Castelo R, Guinney J. GSVA: gene set variation analysis for microarray and RNA-seq data. BMC Bioinformatics. 2013;14:7. doi:10.1186/1471-2105-14-7.

24. Robin X, Turck N, Hainard A, et al. pROC: an open-source package for $\mathrm{R}$ and $\mathrm{S}+$ to analyze and compare ROC curves. $B M C$ Bioinformatics. 2011;12:77. doi:10.1186/1471-2105-12-77. 
25. Yan H, Parsons DW, Jin GL, et al. IDH1 and IDH2 mutations in gliomas. $N$ Engl J Med. 2009;360:765-773. doi:10.1056/NEJMoa0 808710

26. Wang Z, Zhang C, Liu X, et al. Molecular and clinical characterization of PD-L1 expression at transcriptional level via 976 samples of brain glioma. Oncoimmunology. 2016;5:e1196310. doi:10.1080/ 2162402X.2016.1196310.

27. Verhaak RG, Hoadley KA, Purdom E, et al. Integrated genomic analysis identifies clinically relevant subtypes of glioblastoma characterized by abnormalities in PDGFRA, IDH1, EGFR, and NF1. Cancer Cell. 2010;17:98-110. doi:10.1016/j.ccr.2009.12.020.

28. Zheng C, Zheng L, Yoo JK, et al. Landscape of infiltrating T cells in liver cancer revealed by single-cell sequencing. Cell. 2017;169:13421356 e1316. doi:10.1016/j.cell.2017.05.035.

29. Azizi E, Carr AJ, Plitas G, et al. Single-cell map of diverse immune phenotypes in the breast tumor microenvironment. Cell. 2018;174:12931308 e1236. doi:10.1016/j.cell.2018.05.060.

30. Neftel C, Laffy J, Filbin MG, et al. An integrative model of cellular states, plasticity, and genetics for glioblastoma. Cell. 2019;178:835-+. doi:10.1016/j.cell.2019.06.024.

31. Sznol M, Chen L. Antagonist antibodies to PD-1 and B7-H1 (PD-L1) in the treatment of advanced human cancer-response. Clin Cancer Res. 2013;19:5542. doi:10.1158/1078-0432.CCR-13-2234.
32. Lipson EJ, Forde PM, Hammers HJ, Emens LA, Taube JM, Topalian SL. Antagonists of PD-1 and PD-L1 in cancer treatment. Semin Oncol. 2015;42:587-600. doi:10.1053/j.seminoncol.2015.05.013.

33. McGranahan T, Therkelsen KE, Ahmad S, Nagpal S. Current state of immunotherapy for treatment of glioblastoma. Curr Treat Options Oncol. 2019;20:24. doi:10.1007/s11864-019-0619-4.

34. Zhang Y, Chen L. Classification of advanced human cancers based on Tumor Immunity in the MicroEnvironment (TIME) for cancer immunotherapy. JAMA Oncol. 2016;2:1403-1404. doi:10.1001/ jamaoncol.2016.2450.

35. Santegoets KCM, Gielen PR, Bull C, et al. Expression profiling of immune inhibitory Siglecs and their ligands in patients with glioma. Cancer Immunol Immunother. 2019. doi:10.1007/s00262019-02332-w.

36. Li G, Wang Z, Zhang C, et al. Molecular and clinical characterization of TIM-3 in glioma through 1024 samples. Oncoimmunology. 2017;6: e1328339. doi:10.1080/2162402X.2017.1328339.

37. Hamieh M, Dobrin A, Cabriolu A, et al. CAR T cell trogocytosis and cooperative killing regulate tumour antigen escape. Nature. 2019;568:112-116. doi:10.1038/s41586-019-1054-1.

38. Cristescu R, Mogg R, Ayers M, et al. Pan-tumor genomic biomarkers for PD-1 checkpoint blockade-based immunotherapy. Science. 2018;362:eaar3593. doi:10.1126/science.aar3593.
OncoTargets and Therapy

\section{Publish your work in this journal}

OncoTargets and Therapy is an international, peer-reviewed, open access journal focusing on the pathological basis of all cancers, potential targets for therapy and treatment protocols employed to improve the management of cancer patients. The journal also focuses on the impact of management programs and new therapeutic

Submit your manuscript here: https://www.dovepress.com/oncotargets-and-therapy-journal

\section{Dovepress}

agents and protocols on patient perspectives such as quality of life, adherence and satisfaction. The manuscript management system is completely online and includes a very quick and fair peer-review system, which is all easy to use. Visit http://www.dovepress.com/ testimonials.php to read real quotes from published authors. 